\title{
Automated Docking Procedure in Modern Shipping
}

\author{
C. Athanasoglou \\ Dept. of Industrial Design \\ and Production \\ Engineering \\ University of West Attica, \\ Athens, Greece
}

\author{
M. Papoutsidakis \\ Dept. of Industrial Design \\ and Production \\ Engineering \\ University of West Attica, \\ Athens, Greece
}

\author{
D. Papachristos \\ Dept. of Industrial Design \\ and Production \\ Engineering \\ University of West Attica, \\ Athens, Greece
}

\author{
N. Nikitakos \\ Dpt. of Shipping trade \& \\ Transport \\ University of Aegean, \\ Greece
}

\begin{abstract}
The continuing evolution in automatic technologies, the pressing demands and the endless need of gaining time, leads the marine industry to spend more money in order to find out new ways and technologies in the field of automation. Every year new systems appearing in the marine industry and get involve to the development of new building vessels, as to the old constructed.

This document has to do about the presentation of new ideas in the construction of full autonomous vessels. Also trying to forecast the dangerous situations that could be appear during the application of these systems. The presentation including a try to construct a autonomous system of auto docking , constructed by basic electronic equipment that already be used in most marine and industry fields. It will be given extra interesting in the most important parts of a vessel that will be involved in this construction and will be explained the way that these part corporate each other.
\end{abstract}

The mean of Autonomous vessel have a huge range and including a very high intelligent level of technology. In this paper will try to present a very small part of these systems , this is the Autodocking operation.

\section{Keywords}

Autonomous vessels, Auto Docking, Bow thrusters, Wind Indicator, Speed Log, PLC, Rolls Royce, Patent

\section{INTRODUCTION}

The idea of autonomous vessels is not a new idea. Started a lot of years before by using the big range of autonomous system in the vessels. All these systems with the passage of time and the evolution of technologies leads to new ideas about a full autonomous vessel.

Some basic existing applications of autonomous systems in vessels are:

- Internal combustion engine automatic control

- Automatic fire detection systems

- $\quad$ Auto pilot systems

- $\quad$ Automatic doors

- $\quad$ Ais (Automatic Identification systems)

Full autonomous vessels is the new step in modern age. The biggest Maritime companies in the world has already start before many years the first attempts in order to construct the first full autonomous vessel so they will be ready and full involved in the age of technological evolution.

These companies are Kongsberg Gruppen S.A that in corporation with Yara ASA planning the construction of the first container vessel in the world. This project is about to start in the beginning of 2019 .

Rolls Royce also has also get involved in this, the industrial center of research located in Finland (AAWA) started planning these projects years ago. The answers that trying to find are about, What technology is needed and how can it be best combined to allow a vessel to operate autonomously, miles from shore? How can an autonomous vessel be made at least as safe as existing ships, what new risks will it face and how can they be mitigated? What will be the incentive for owners and operators to invest in autonomous vessels? Are autonomous ships legal and who is liable in the event of an accident?

Funded by Tekes (Finnish Funding Agency for Technology and Innovation) the project brings together leading international maritime businesses and top Finnish universities (details of which can be found on the back page). The project has examined the current state of the maritime industry and what can be learnt from other industries - from aviation's drones and driverless cars to the smartphone. It is now exploring the current state of understanding of the technological, safety, legal and economic aspects of remote and autonomous operation. The results will inform the specification and preliminary designs for a proof of concept demonstrator by the end of 2017 and a remote-controlled ship in commercial use by the end of the decade.

The need is to develop a set of electronic senses that inform an electronic brain and allow the vessel to navigate safely and avoid collisions. The AAWA Project is exploring three areas:

1. Sensor fusion Sensor technology is well developed and found in many forms of autonomous vehicle operation, most notably cars where competing developers have prioritized differing technologies. The AAWA project has explored the contribution different sensor technologies make in providing a vessel or its remote operators with an accurate perspective on the vessel's surroundings at all times and in all conditions. Looking at different types of radars, high definition visual cameras, thermal imaging and LIDAR the project has concluded fusing multiple sensor inputs provides the best results. The main question is not can this be done? It is how to combine these technologies in the most cost-efficient way considering the challenges of the maritime environment. Finding the optimum way to combine the different sensor technologies in a range of operating and climatic conditions will be the subject of a series of tests at sea in 2016 .

2. Control algorithms Navigation and collision avoidance will be particularly important for remote and autonomous ships, allowing them to decide what action to take in the light of sensory information received. The decision algorithms behind this need perfecting, as it requires an interpretation of 
maritime rules and regulations. This leads to interpretation challenges for the programmer. The development of control algorithms for autonomous vessels will be a gradual and iterative process and subject to extensive testing and simulation

3. Communication and connectivity Autonomous vessels will still need human input from land, making connectivity between the ship and the crew crucial. Such communication will need to be bidirectional, accurate, scalable and supported by multiple systems - creating redundancy and minimizing risk. Sufficient communication link capacity for ship sensor monitoring and remote control, when necessary, has to be guaranteed. The project is exploring how to combine existing communication technologies in an optimum way for autonomous ship control. We have created a simulated autonomous ship control system which will be connected to a satellite communications link as well as land-based systems. This will allow us to explore the behavior of the complete system

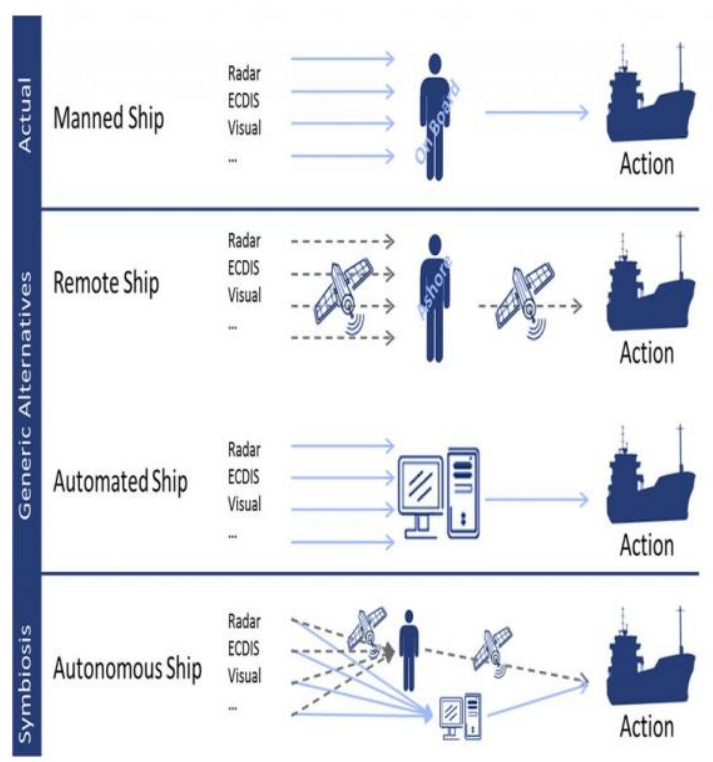

Fig 1: Types of ships

Munin also is another big company involved in these systems. As per Munin, Autonomous vehicles are already state-of-theart in many land based transport modes. There exist several examples of automated subways, self-driving intralogistics vehicles or automated guided vehicles (AGV) on modern container terminals. There are also very wide-ranging approaches of autonomous control concepts in modern aviation.Consequently, autonomy is also seen as a possibility for maritime transport to meet today's and tomorrow's competitiveness, safety and sustainability challenges. According to Waterborne TP an autonomous ship is described as:

Next generation modular control systems and communications technology will enable wireless monitoring and control functions both on and off board. These will include advanced decision support systems to provide a capability to operate ships remotely under semi or fully autonomous control.

This description implies two generic alternatives that are combined in an autonomous ship (see also figure below): the remote ship where the tasks of operating the ship are performed via a remote control mechanism e.g. by a shore based human operator and

the automated ship where advanced decision support systems on board undertake all the operational decisions independently without intervention of a human operator.

Within MUNIN's idea of an autonomous and unmanned vessel both generic alternatives will be combined in a holistic concept. Developing and validating a suitable mixture of remote and automated technology for ships will be the core task of the MUNIN project. The onboard decision system approach that is implemented in MUNIN may be illustrated as in the figure below.

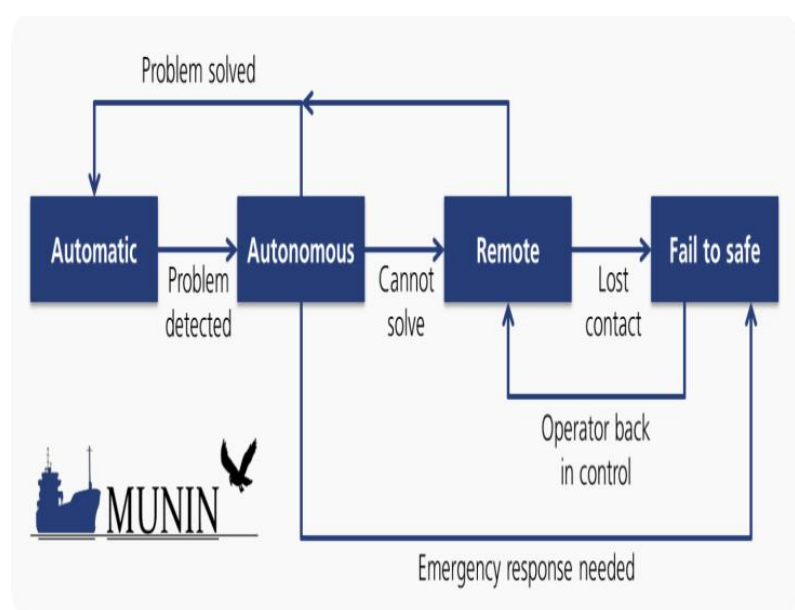

Fig 2:

MUNIN would normally rely on automatic and fully deterministic control functions to run the ship. However, various sensor systems will be needed to detect problematic situations such as unexpected objects in the sea, dangerous weather conditions or danger of collision. If an unexpected situation occurs, an autonomous control module will be invoked trying to remedy the situation within its given constraints. If the system cannot achieve this, it will request support from a remote operator or start a fail-to-safe procedure if the operator is not available. Properly implemented, this type of autonomy will reduce the need for human supervision while maintaining a high and well defined level of safety. However, a major challenge will be to device sensor systems so that all relevant dangerous situations are reliably detected and appropriately acted upon.

\section{METHODOLOGY}

The methodology of our attempt to introduce a automatic docking system is based in a combination of vessel's system like propulsion systems, bow thruster systems and navigation systems. This combination will communicate each other via a plc system, in that case a plc Siemens Logo 8 that is programmed in FBD language. The basic panel that the programming logic based appearing in the next board. Figure 3 


\begin{tabular}{|c|}
\hline Level Description \\
\hline 10 The computer does everything autonomously, ignores human \\
\hline 9 The computer informes human only ifit (the computer) decides so \\
\hline 8 The computer informes human only if asked \\
\hline 7 The computer executes automatically, when neccssary informing human \\
\hline 6 The computer allows human a restricted time to veto before automatic execution \\
\hline 5 The computer executes the suggested action if human approves \\
\hline 4 Computer suggests single alternative \\
\hline 3 Computer narrows aleternatives down to a few \\
\hline 2 The computer offers a complete set of decision alternatives \\
\hline 1 The computer offers no assistance, human in charge of all decisions and actions \\
\hline
\end{tabular}

Fig 3: Level Description

This panel appears the common logic and the priority of each system be involved in the autodocking application. Determines the dynamic action and the independence role of each one. In case that something is going wrong the system surrenders the control to human operator.

In general the autodocking system use :

1. Bow thruster operation, is electromechanical system of propeller that located in the bow of the vessel. In operation mode the operator orders the side to turn the vessel and the propeller do that job.

2. Wind indicator, is the sensor that counting the direction and the volume of the wind. In our system is responsible as a factor of the sensitivity. If the wind direction is in the same direction of location moving the vessel, bow thrusters will operate with easier volume than the opposite direction.

3. Speed log, is the sensor about measuring the speed and the direction of the water and as like the anemometer (wind indicator) counts the factor of water as another factor of sensitivity.

4. Distance sensors, is one of the most important part of the application. Is the sensor that counting the distance between the vessel and the jetty. Must used sensors with high sensitivity and high intelligent systems for this scope.

\section{DESCRIPTION}

The idea is based in the construction of a PID controller that combinate all the previous referred systems. When the vessel arranges the port and is going to proceed the jetty. Before the auto docking application get involve, distance sensors must be placed in specific locations at the side of docking. This could be performed by vessel's crew because sensors have no big size and weight, also is easy enough in future to construct an automatic procedure for that. When all parameters are ready, the vessel is stopped and the sensor in the competent

\section{CONCLUSION}

During the research about autonomous vessels, realized that there are many attempts many years ago, by big companies in the maritime industry to construct the completely fully autonomous vessel. Last years the evolution of technology, gave them the chance to perform huge steps in achievement of this challenge. Although the high technologic level and the high amount is spending every day for that purpose, this project is still experimental. This is due to high level of difficulties that must be faced and to the high safety risks that position, captain push the start button and the procedure starts. Bow thrusters operate until vessel reach the in the normal distance from the jetty and then vessel is ready to be secured.

The system is extremely sensitive and dangerous. In case of wrong usage it is possible damage the vessel , even more cause serious accidents. For that reason there are safety rules that must be follow. These are:

1. All involved equipment in the system must be duplicated, in order of malfunction the damaged equipment must be replaced automatic by the same unit immediately.

2. In any case must be placed emergency controller that delivering the operation mode to the operator.

3. Indication leds must be appearing the procedure and any errors or alarms in the system continuously.

4. Continuously supervision by authorized person must be performed.

5. Tactical survey by authorized technicians and class surveyors must be performed

6. Every three months must be perform sore base maintenance.

7. Installed equipment must be agree with IMO regulations.

8. New installed systems must be applied only to new building vessels.

9. CCTV systems must be applied and recording the procedure.

10. The system must be completely recorded by the Voyage Data Recorder (VDR).

Criteria must me issue for the propriate functionality of Auto Docking system

1. Before activate the system by the start button, the vessel must be fully stopped.

2. Plc program is responsible for the control of this parameter. Checking continuously the values from engine, thrusters, wind indicator and speed log for this purpose.

3. The distance between the jetty and the vessel must not be over 27 meters, that is why the installed distance sensors have rage maximum 30 meters.

4. Bow thrusters must be deactivated when the application starts

5. Anemometer and speedlog must be in good working condition because are responsibly for the sensitivity of the PID controller.

must be taken before be sure to apply it in the 'vessel's world ". The technical knowledge is still in low level for the implementation such a project and the amounts needed are still huge . Even if say that all these problems could be solved soon, the safety factor is always the most important reason for further researching. It is possible that the next years we will see the first autonomous vessels appearing over the sea, but the human factor is very difficult to be ignored and at least the next 15 years will be present on every autonomous vessel. The reason of that is the biggest deference between machine and human, the initiative factor. 


\section{ACKNOWLEDGMENTS}

All authors would like to thank the University of West Attica and specifically the Post Graduate Program of Studies (MSc) "New Technologies in Shipping and Transport", for the financial support provided to them to undertake this research project.

\section{REFERENCES}

[1] https://elgrecoshipyards.blogspot.com/2015/06/ei-bowstern-thruster.html

[2] https://www.plcacademy.com/function-block-diagramprogramming/
[3] https://www.youtube.com/results?search_query=full+aut o+ship

[4] https://patents.google.com/patent/US6273771B1/en

[5] https://patents.google.com/patent/US8622778B2/en

[6] https://patents.google.com/patent/WO2012068425A1/en

[7] https://www.rolls-royce.com/ /media/Files/R/RollsRoyce/documents/customers/marine/ship-intel/rr-shipintel-aawa-8pg.pdf

[8] http://www.unmanned-ship.org/munin/about/theautonomus-ship/ 